\title{
Surface properties and wear performances of siloxane-hydrogel contact lenses
}

Michela Bettuelli, Silvia Trabattoni, Matteo Fagnola, Silvia Tavazzi*, Laura Introzzi, Stefano Farris

M. Bettuelli, Dr S. Trabattoni, M. Fagnola, Dr S. Tavazzi*

University of Milano Bicocca, Department of Materials Science, Via Cozzi 53, I-20125

Milano (Italy)

Dr L. Introzzi, Dr S. Farris

University of Milan, Department of Food, Environmental and Nutritional Sciences

(DeFENS)-Packaging Lab, Via Celoria 2, I-20133 Milano (Italy)

E-mail: silvia.tavazzi@unimib.it

Fax: +390264485400

Running Heads: surface properties of siloxane-hydrogel contact lenses and OSDI 


\section{ABSTRACT}

The low surface roughness of disposable contact lenses made of a new siloxane-hydrogel loaded with hyaluronic acid is reported, as studied by atomic force microscopy (AFM). Before the wear, the surface is characterized by out-of-plane and sharp structures, with maximum height of about $10 \mathrm{~nm}$. After a wear of eight hours, evidence of two typical morphologies is provided and discussed. One morphology (sharp-type) has a similar aspect as the unworn lenses with a slight increase of both the height and the number of the sharp peaks. The other morphology (smooth-type) is characterized by troughs and bumpy structures. Wettability and clinical performances are also discussed, the latter deduced by the OcularSurface-Disease Index (OSDI). The main finding arising from this work is the indication of correlation between the change of the OSDI before and after wear and the lens surface characteristics obtained by AFM.

\section{Keywords:}

- New siloxane-hydrogel material;

- Atomic Force Microscopy (AFM);

- Disposable contact lenses;

- Wettability;

- Wear of contact lenses and Ocular Surface Disease Index (OSDI). 


\section{Introduction}

In the last years, the advent of siloxane-hydrogel ( $\mathrm{Si}-\mathrm{Hy})$ materials brought a revolutionary advance in the contact lens field. ${ }^{[1]}$ The main advantage of these materials is the ability of siloxane moieties to promote the transport of oxygen and carbon dioxide across the lens. As a consequence, the use of Si-Hy has shifted the topics of research from hypoxic complication, removed by high gas permeability, to hydration and surface properties, due to the typical relatively poor wettability of Si-Hy. To enhance wettability, which is typically measured by the contact-angle technique, different approaches have been pursued by researchers and by manufacturers. One strategy is based on surface treatments, plasma coatings, or plasma oxidation; ${ }^{[2-4]}$ another way is the inclusion of an internal wetting agent. ${ }^{[5,6]}$ Another important aspect is the hydration of the lenses, which typically ranges from $20 \%$ to $50 \%$ for lenses made of Si-Hy materials. ${ }^{[7]}$ A promising approach to maintain a high level of hydration is the inclusion of hyaluronic acid (HA), which is a natural anionic polyelectrolyte present in many tissues of the human body; it has wide applications in medicine and biotechnology and it has also been proposed as a remedy for the dry-eye syndrome. ${ }^{[8-16]}$ Besides measurements of hydration and surface contact angle, atomic force microscopy (AFM) has also been used in recent years to characterize contact lens surface both in liquid and anhydrous conditions. ${ }^{[17-22]}$ Changes of surface roughness after wear have been studied for some hydrogels and $\mathrm{Si}-\mathrm{Hy}$ based contact lenses. Indeed, the surface of a contact lens is in direct contact with the tear film and the conjunctiva, thereby investigating the surface properties turns to be a crucial point due to the involved clinical implications. Different tests can be used to evaluate the clinical performances, such as staining of the ocular surface, Tear film Break-Up-Time (TBUT), Schirmer test, Ocular-Surface-Disease Index (OSDI), the latter being used to have information on the ocular symptomatology. ${ }^{[23]}$ For example, during the wear of contact lenses, the OSDI has been reported to be correlated to dry-eye symptoms and to mucin fragmentation on the surface of the lens. ${ }^{[24-26]}$ 
Based on the aspects discussed above, this paper is aimed at presenting (i) some properties of both new and worn contact lenses made of a new $\mathrm{Si}-\mathrm{Hy}$ polymer loaded with $\mathrm{HA}$, including the surface properties deduced by AFM and contact-angle measurements, (ii) relevant differences observed in the surface morphology of worn lenses, and (iii) the correlations that have been found among the surface physical properties and the clinical performances of the lenses, the latter described by the ocular-surface-disease index (OSDI).

\section{Materials and Methods}

The HA-containing ${ }^{[27]}$ contact lenses used in this work (St. Shine Optical Co., LTD, Taiwan) have been kindly supplied by Safilens (Italy). Some characteristics of the lenses (power ranging from $-1.00 \mathrm{D}$ to $-4.00 \mathrm{D}$ ) are reported in Table I. A limited number of contact lenses of the same material, but without HA, has been supplied by Safilens (Italy), only for chemical and physical analyses. These lenses were not used for wear. They were loaded with $\mathrm{HA}^{[27,28]}$ in our laboratory in a water solution of HA $(2.0 \mathrm{mg} / \mathrm{ml})$ and the content of HA in each lens has been evaluated to be of few tens of $\mu \mathrm{g}$. Some characteristics of the used HA, such as the average molecular weight and the polydispersity index are reported in Table S-I of the Supporting Information.

Surface morphology has been studied by using the atomic force microscope (AFM) Nanoscope V MultiMode (Veeco). Measurements have been performed in air in tapping mode using RTESP silicon tips (Veeco, spring constant 20-80 N/m, resonance frequency 287-346 $\mathrm{kHz}$ ). The lenses have been extracted from the blister with tweezers, rinsed three times in deionized water to remove residual packaging solution, and then mounted on a semispherical glass holder mimicking the lens curvature radius. Images of the front surface of the lenses have been recorded with the resolution of $512 \times 512$ pixels and corrected by zero or higher order polynomial background filters depending on the scan size. Root-mean-square roughness 
$\left(\mathrm{R}_{\mathrm{rms}}\right)$, skewness $\left(\mathrm{R}_{\mathrm{sk}}\right)$ and kurtosis $\left(\mathrm{R}_{\mathrm{ku}}\right)$ have been measured for all the analyzed lenses: 10 lenses denoted as $\mathrm{CL}_{\text {rins, }}$ taken from the blister and rinsed with deionized water; 5 lenses denoted as $\mathrm{CL}_{\mathrm{ss}}$, preserved 12 hours in saline solution and rinsed with deionized water; 22

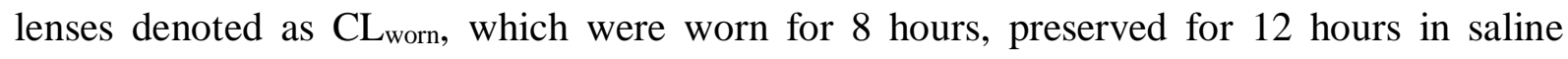
solution, and rinsed in deionized water (for the worn lenses, 4 images have been eliminated from the AFM analysis due to AFM experimental artifacts); 5 lenses purchased from Johnson\&Johnson (Galyfilcon A, Acuvue Advance, -3.00D) denoted as $\mathrm{CL}_{\text {Galy. }} \mathrm{R}_{\mathrm{sk}}$ is a measure of the asymmetry of the height value distribution with respect to the mean value, showing positive values when the distribution exhibits a tail on its right side and negative values when the tail is on the left side. Therefore, the $\mathrm{R}_{\mathrm{sk}}$ value can be related to the predominance of peaks $\left(R_{s k}>0\right)$ or to the abundance of troughs $\left(R_{s k}<0\right) . R_{k u}$ is related to the shape of the probability distribution, showing values higher than 3 for sharp distributions and values lower than 3 for bumpy shaped distributions. In this case, $R_{k u}$ value can be related to the abundance of sharp structures $\left(\mathrm{R}_{\mathrm{ku}}>3\right)$ instead of bumpy structures $\left(\mathrm{R}_{\mathrm{ku}}<3\right)$. For the AFM analyses, it has not been possible to acquire proper images on lenses taken from the blister without rinsing them in water, because of the presence of salts, causing strong interactions with the AFM tip.

Contact angle analyses have been performed using an optical contact-angle apparatus (OCA 15 Plus - Data Physics Instruments GmbH, Filderstadt, Germany) equipped with a video measuring system with a high resolution CCD camera and a high performance digitizing adapter. The software SCA 20 (Data Physics Instruments GmbH, Filderstadt, Germany) has been used for data acquisition. Contact angles determinations have been carried out on four different batches: lenses directly taken from the blister $\left(\mathrm{CL}_{\mathrm{bl}}\right), \mathrm{CL}_{\text {rins }}, \mathrm{CL}_{\mathrm{ss}}$, and $\mathrm{CL}_{\text {worn. }}$ The procedure behind each sample preparation before analysis has been carefully standardized. In particular, each sample has been first blotted to remove excess liquid, mounted on the same custom-made glass holder as seen for the AFM analysis, and then blotted again by means of a 
microfiber cloth, in order to further remove the residual liquid throughout the lens top surface. ${ }^{[29]}$ The worn lenses were stored after wear in saline solution for 12 hours before the contact-angle measurements. Measurements have been run according to the sessile drop method, i.e. gently dropping a droplet of $(3.0 \pm 0.4) \mu 1$ of Milli-Q water $(18.3 \mathrm{M} \Omega \mathrm{cm})$ onto the surface of the contact lens, at $(20 \pm 1)^{\circ} \mathrm{C}$ and $(35 \pm 5) \%$ relative humidity. All droplets have been released from a height of $1 \mathrm{~cm}$ above the surface to ensure consistency between each measurement. The contact angle value has been taken $10 \mathrm{~s}$ after the droplet placement, to reach the equilibrium at the solid/liquid interface. To this purpose, a semi-automatic image analysis procedure has been adopted. For each lens type, the final contact angle value and its standard deviation have been obtained from at least five measurements taken from different replicates. This also allowed to monitor the repeatability of the results. The surface tension of the blister packaging solution has also been assessed using the same apparatus as above, coupled with the SCA 22 software, according to the pendant drop method.

Our group of wearers was made of 11 subjects aged $23 \pm 3$ years ( 5 females and 6 males). Some subjects repeated the wear twice at the distance of weeks. 7 subjects are habitual wearers of contact lenses and 4 subjects are occasional wearers. They have been instructed to wear contact lenses for 8 hours, after 3 days without any contact lens wear. Before the wear, the ocular condition was evaluated and graded by Efron Grading Scales; the horizontal corneal diameter, the eyelid aperture, and the blink frequency have been measured; the eye topography has been collected, the tear film stability was assessed using tear film break time (TBUT) and non-invasive TBUT; the lower Tear Meniscus Height (TMH) was measured using a slit-lamp biomicroscope without fluorescein to evaluate the tear volume. Before the wear, the wearers have been asked to provide information about their eye symptoms and comfort during the day before the wear, filling up a questionnaire. ${ }^{[23]}$ The same questionnaire was used after wear to provide information on the eye symptoms during the 8 hours of wear. From each questionnaire, the OSDI score was deduced. As reported in the literature, ${ }^{[23]}$ the 
OSDI has been validated to be a reliable instrument for measuring the ocular surface disease when the questions are referred to a period of one week. We have used this index even if, in our case, the questions were referred to a period of one day, either the day before the wear or the day of wear of the contact lenses. The single score ranges from 0 to 4 (never, rarely, sometimes, often, always) and is expressed for eight possibly-felt ocular sensations and for four possibly-experienced problems in various activities. In some cases, the answers to some questions were not given. This possibility is taken into consideration in the calculation of the score. ${ }^{[23]}$ The sum of all scores is defined as the OSDI and it ranges from 0 (maximum comfort) to 100 (maximum discomfort). All subjects provided informed consent.

Statistical significance of differences among measured quantities for the different types of lenses has been evaluated by Student's t statistic (t-tests). Significant difference is considered to occur if the probability value $(p)$ is lower than 0.05 . When studying the correlation between measured quantities, the linear correlation coefficient $\mathrm{R}$ between the two groups has been calculated. Significant correlation is considered to occur if the correlation probability $\left(p_{\mathrm{c}}\right)$ is lower than 0.05 .

\section{Results}

Our first analysis has concerned the surface morphological characteristics observed by AFM. As shown in Figure 1, the surface morphology of $\mathrm{CL}_{\text {rins }}$ lenses taken from the blister and rinsed in deionized water appears regular and relatively flat. Figure 2 shows the AFM images taken on $\mathrm{CL}_{\mathrm{ss}}$ lenses. Before wear, the main morphological characteristics are similar as for the $\mathrm{CL}_{\text {rins }}$ lenses. Changes were observed after 8-hour wear, as shown in Figures 3 and 4. In these cases, we have observed two typical morphologies, with different characteristics. One type of samples (Figure 3) shows structures which are out-of-plane and sharp, with maximum height equal to about $30 \mathrm{~nm}$, to be compared with $10 \mathrm{~nm}$ for the unworn $\mathrm{CL}_{\text {rins }}$ lenses (Figure 
1a). These observations suggested us to denote this morphology as sharp-type. Considering the total number of examined worn lenses, this morphology occurred in the $60 \%$ of the cases. Figure 4 represents a totally different morphology, characterized by troughs and bumpy structures. It was indicated as the smooth-type morphology and it was found in the other $40 \%$ of the wearers. Overall, the AFM morphology of the sharp-type lenses is similar to that of the unworn lenses, while the smooth-type lenses show a very different aspect. This is clearly evident by comparing Figs. 1, 3, and 4. The differences between the two morphologies of the worn lenses are also evident in the 3D images in Figure 5.

Quantitative results arising from the AFM analyses are summarized in Table II. First of all, we underline that the coefficient of variation (defined as the ratio between the standard deviation and the mean) is typically relatively high, ranging from about 0.2 up to 1 . These large coefficients derive from the spread of the data, combined in some cases to the relatively low mean value. After averaging the results of several samples, the roughness of the $\mathrm{CL}_{\text {rins }}$ lenses on $(5 \mu \mathrm{m} \times 5 \mu \mathrm{m})$ scan size has been found equal to $(1.5 \pm 1.3) \mathrm{nm}$. This value has been compared with that recorded for another Si-Hy material without surface treatments, namely Galyfilcon A, showing similar water content and oxygen transmission level as the material under investigation. For Galyfilcon $A$, the roughness deduced from $(5 \mu \mathrm{m} \times 5 \mu \mathrm{m})$ images has been measured to be $(1.6 \pm 0.3) \mathrm{nm}$, similarly as reported in the literature. ${ }^{[22]}$ The difference among the roughness of these two types of lenses is not statistically significant $(p=$ 0.8252 , Table S-II). Also the $(10 \mu \mathrm{m} \times 10 \mu \mathrm{m})$ roughness of Galyfilcon A $((1.9 \pm 0.4) \mathrm{nm})$ is comparable with the measured value $((2.6 \pm 2.2) \mathrm{nm})$ for our Si-Hy polymer $(p=0.3429$, Table S-II). Indeed, both materials have a very regular and homogenous surface. However, Galyfilcon A presents a porous morphology, as shown in Figure S-1 of the Supporting Information. For the $\mathrm{CL}_{\mathrm{ss}}$ lenses, the measured AFM roughness is similar as for the $\mathrm{CL}_{\text {rins }}$ lenses $\left(p=0.8032\right.$, Table S-III). For the worn lenses, $\mathrm{R}_{\mathrm{rms}}$ is larger for the sharp-type worn lenses than for the smooth-type ones, with $p$ values indicating statistical differences $(p=$ 
0.0170, Table S-IV), with acceptable coefficients of variation, notwithstanding the reduced number of measured samples. The sharp-type lenses also show a larger $\mathrm{R}_{\mathrm{rms}}$ than the unworn $\mathrm{CL}_{\text {rins }}$ lenses, with statistical significance $(p=0.0275$, Table $\mathrm{S}-\mathrm{V})$.

As far as the values of $R_{s k}$ and $R_{k u}$ are concerned, the comparison between $\mathrm{CL}_{\text {rins }}$ and $\mathrm{CL}_{\mathrm{Galy}}$ confirms their different morphology, notwithstanding the similar $\mathrm{R}_{\mathrm{rms}}$. Indeed, as reported in Table $\mathrm{S}$-II, the $\mathbf{R}_{\mathrm{sk}}$ values for these two lenses are statistically different $(p=0.0145)$ and $\mathrm{R}_{\mathrm{ku}}$ shows a tendency to be different $(p=0.1010$, Table S-II), within the limit of the very large coefficients of variation. The skewness $R_{\text {sk }}$ for the $C_{\text {rins }}$ lenses is positive $(2.8 \pm 2.4)$ and indicates that the structures which dominate the surface are out-of-plane. The kurtosis $\left(\mathrm{R}_{\mathrm{ku}}\right.$ >3) suggests the presence of sharp structures. For Galyfilcon A, $R_{\text {sk }}$ is close to zero, indicating that the height distribution is symmetric with respect to its mean value. The $\mathrm{CL}_{\mathrm{ss}}$ lenses show sharp and out-of-plane structures $\left(\mathrm{R}_{\mathrm{ku}}>3, \mathrm{R}_{\mathrm{sk}}>0\right)$, with no significant differences with respect to the $\mathrm{CL}_{\text {rins }}$, lenses ( $p=0.3804$ and $p=8162$ for $\mathrm{R}_{\mathrm{sk}}$ and $\mathrm{R}_{\mathrm{ku}}$, Table $\mathrm{S}-\mathrm{III}$ ). For the worn lenses, $R_{s k}$ and $R_{k u}$ are larger for the sharp-type worn lenses than for the smooth-type ones ( $p=0.0001$ and $p=0.0082$, respectively, Table S-IV), but the coefficients of variation are very large. The sharp-type lenses also show a larger $\mathrm{R}_{\mathrm{sk}}$ compared with the unworn $\mathrm{CL}_{\text {rins }}$ lenses (with no clear statistical significance, but indicative since $p=0.0769$, Table S-V), while $\mathrm{R}_{\mathrm{ku}}$ is comparable, as confirmed by the very high $p$ value $(0.9493)$ in Table $\mathrm{S}-\mathrm{V}$ of the Supporting Information. This indicates that the sharp-type lenses are characterized by sharp peaks, similarly as $\mathrm{CL}_{\text {rins, }}$, but the wear has determined an increase of the number of these thin structures. On the contrary, the $R_{s k}$ and $R_{k u}$ values of the smooth-type lenses can be reasonably considered different with respect to the $\mathrm{CL}_{\text {rins, }}$ with no clear statistical significance, but indicative (Table $\mathrm{S}-\mathrm{V}$ ). This indicates that the modifications of the surface have produced larger area with comparable heights, instead of sharp and relatively high peaks. We have also evaluated the lens wettability by contact-angle measurements before and after wear (as can be seen by the images displayed in Figure 6). In this case, it has also been 
possible to analyze lenses directly taken from the blister without any rinsing $\left(\mathrm{CL}_{\mathrm{bl}}\right)$, in contrast to AFM. The mean values and the standard deviations for the four types of lenses are reported in Table III. The repeatability of the results is supported by the coefficient of variation, which is found to be within few $\%$ (8\% for $\mathrm{CL}_{\mathrm{bl}}, 7 \%$ for $\mathrm{CL}_{\mathrm{ss}}, 4 \%$ for $\mathrm{CL}_{\text {rins }}$ and $\left.\mathrm{CL}_{\text {worn }}\right)$.

The measured contact-angle for the $\mathrm{CL}_{\mathrm{bl}}$ lenses is statistically very different with respect to all the other types of lenses taken into consideration, as observed in Table III, as confirmed by the $p$ values in Table S-VI of the Supporting Information, and as it can also be seen by the images displayed in Figure 6. On the contrary, when comparing the other lenses, no clear evidences of statistically significant differences are found. Only the $p$ values (Table S-VI) for $\mathrm{CL}_{\text {worn }} / \mathrm{CL}_{\text {rins }}$ and $\mathrm{CL}_{\text {worn }} / \mathrm{CL}_{\mathrm{ss}}$ are indicative of possible differences, which are however relatively small.

The physical characterization has been accompanied by a clinical study in terms of symptomatology declared by the wearers through the OSDI. The results are reported in Table IV , corresponding to the wearer symptomatology both before and after the wear. Considering all wearers, the mean of the OSDI after wear was equal to $7.8 \pm 5.6$ (not reported in Table IV), to be compared with $4.6 \pm 3.4$ before wear $(p=0.0142)$. In general, the wearers declared a good eye condition after 8-hour wear with some slight differences among them. To investigate in depth a possible relationship between surface lens properties and clinical performances, the change in the OSDI before and after wear $(\Delta(\mathrm{OSDI})$, Table IV) and the parameters deduced by the AFM analysis after the wear are compared in Figure 7 for each wearer. Black diamonds and red squares indicate the data corresponding to the different observed morphologies. The numbers in the labels indicate each single wearer. In some cases, AFM images were not taken into consideration for the analysis because affected by experimental artifacts (images of poor quality), so that few numbers are lacking; other numbers are repeated twice (one for each eye), other numbers are present only once. First of 
all, we notice that the observed morphology for the right eye is the same as the morphology corresponding to the left eye of the same wearer after the same period of wear. The $R_{r m s}, R_{s k}$, and $\mathrm{R}_{\mathrm{ku}}$ values for the right and left eyes of the same wearer are also in reasonable agreement, their difference being usually relatively small compared to the difference with the results for other wearers. The difference between the $\mathrm{R}_{\mathrm{rms}}$ for the two eyes of the same wearer ranges from $4.8 \%$ (wearer 7 ) to $26.6 \%$ (wearer 8 ) with respect to the wearer mean value. Only in one case, a large difference has been found (79.3\% for wearer 9 with sharp morphology). Similar considerations can be done for $\mathrm{R}_{\mathrm{sk}}$ and $\mathrm{R}_{\mathrm{ku}}$, as can be reasonably understood from Fig. 7 . We also underline that, for two wearers (6 and 9), the AFM analysis has been performed after two different periods of wear. The same morphology has been observed twice for the wearer 6 , whilst the wearer 9 showed the smooth morphology the first time and the sharp-type one after the second period of wear. From Fig. 7, we notice that there is a non-negligible correlation among the $\Delta(\mathrm{OSDI})$ and the AFM results. The first panel indicates a correlation between $\Delta(\mathrm{OSDI})$ and $\mathrm{R}_{\mathrm{rms}}\left(\mathrm{R}=0.63, p_{\mathrm{c}}=0.0049\right)$. The worst comfort is correlated to the largest roughness. A good correlation has also been found among $\Delta(\mathrm{OSDI})$ and $\mathrm{R}_{\mathrm{sk}}(\mathrm{R}=0.74$, $\left.p_{\mathrm{c}}=0.0007\right)$.

Similarly as Fig. 7, Figure 8 shows the measured contact angle $\theta$ as a function of the corresponding $\Delta(\mathrm{OSDI})$. Evidence of correlation is found $\left(\mathrm{R}=0.78, p_{\mathrm{c}}=0.0009\right)$. As far as the contact-angle data are concerned, it was not possible to split the results into the two smoothtype and sharp-type groups.

\section{Discussion}

Taking into consideration the AFM observations, we underline that the unworn lenses have a homogenous morphology without micro-pores or stripes and a relatively low value of 
roughness, comparable to the roughness of other Si-Hy lenses without surface treatments (i.e. Galyfilcon A). The low roughness of the unworn lenses and the non-ionicity of the material lead us to expect a low content of protein lachrymal residues after wear. Indeed, after 8 hours of wear, no large structures are detected on the lens surface. Moreover, the typical protein absorption band at $280 \mathrm{~nm}$ was not detected in the UV-visible absorption spectrum of the worn lenses (Supporting Information), thus indicating no protein residues within the sensitivity limit of the UV-visible technique. A detailed investigation of possible lipid deposits would be interesting. However, this aspect has not been investigated in this context. Indeed, lipid deposits on the surface have been demonstrated to determine a decrease of the contact angle ${ }^{[30]}$, which has not been measured on our lenses after wear. We focused the attention on the surface morphology and roughness. The increase in roughness for the sharptype morphology after 8 hours of wear could be unworthy for a not-daily lens, but previous studies $^{[31]}$ have demonstrated that a significant changing in roughness often occurs after only 20 minutes of wear and it shows a slight increase during the remaining wearing period. At the same time, for some wearers a new morphology appears (the smooth type). In this latter case, the surface shows a new structure with voids, that we tentatively explain as caused by a loss of materials. The observed modifications after wear for our smooth-type lenses are very similar to the ones reported for other lenses (Acuvue) before and after testing them with an abrasion device (reported by Goldberg, et al. ${ }^{[32]}$ ). In this latter case, AFM and scanning electron microscopy analyses showed that the surfaces of both worn lenses and lenses tested with the abrasion device were significantly altered with ridges, valleys, and formation of elevations, depressions, and distinct grooves, due to damage of the surface. These authors considered a period of wear of some weeks and they concluded that the surfaces of their contact lenses were subjected to abrasive damage by the cornea during wear. This damage was considered to be an initial mechanism event, followed by formation of interfacial debris and deposits. In our case, for $40 \%$ of wearers, we found this effect after 8 hours of wear. The 
effect has been observed on the front surface of the lens, thus being attributable to abrasion by the eyelid. However, the origin of these surface damage requires further analysis. It could be related to the lens fitting, but we believe that the wear conditions (physiological and/or environmental) must play a pivotal role. Indeed, in our study, it may occur that the same wearer presents either one or the other type of morphology in different wear occasions. For example, this occurred for subject 9, who showed a type of morphology after 8-hour wear and, at a distance of few weeks, the other type of morphology.

As far as the contact-angle measurements are concerned, we have measured a higher contact angle after rinsing the lens, this increase after washing being also reported in the literature ${ }^{[29]}$ by Maldonado-Codina and Morgan, who observed an increase from $66^{\circ}$ to $96^{\circ}$ for Galyfilcon A material. As pointed out by these authors, this can be due to the removal of surface active agents upon washing. We believe that the increase of the contact angle after rinsing is due to the removal from the surface of the lenses of active hydrophilic agents previously added in order to enhance compatibility at the lens/eye interface by supporting a stable tear layer in the eye. However, we are prone to exclude that this agent is HA. Indeed, in a previous study we loaded hydrogel contact lenses with HA and we found that, after 5 days of immersion in $1 \mathrm{ml}$ of deionized water at $24{ }^{\circ} \mathrm{C}$, the HA released by the lens was lower than $2.0 \mu \mathrm{g} / \mathrm{ml} .{ }^{[28]}$ The same effect arising from rinsing has been apparently achieved by soaking the contact lenses in a saline solution. Also in this case, the increase of the contact angle is presumably due to the disappearance of hydrophilic agents from the lens surface. It seems that the wear for 8 hours has induced a further increase in the final contact angle with respect to $\mathrm{CL}_{\text {rins }}$ and $\mathrm{CL}_{\mathrm{ss}}$ lenses, although with relatively poor statistical significance. Based on the data reported in the literature, we stress that an increase of the contact angle is expected to be incompatible with lipid deposits on the surface. ${ }^{[30]}$ Our tentative explanation of the increase of the contact angle is the modification of the surface morphology after wear. As also discussed in the literature ${ }^{[33-35]}$ the morphology, the pattern, and the roughness of a surface are relevant 
aspects in dictating the ultimate wettability properties (i.e. the contact angle values). Moreover, loss of moisture at the lens/air interface should be taken into account.

The results of the physical characterization (AFM results and contact angles) have been compared with the results of the clinical study. The OSDI values and their variations $\Delta(\mathrm{OSDI})$ are all relatively low, thus indicating a general good comfort. Within the small changes, indications of correlation between the AFM parameters and $\Delta(\mathrm{OSDI})$ are found, similarly as also found between the contact angle and $\Delta(\mathrm{OSDI})$. We also notice that the two morphologies are distinguishable in Fig. 7. With the exception of the wearer 2, the points for the smooth morphology (black diamonds) typically correspond to both lower AFM values and lower $\Delta(\mathrm{OSDI})$ values than the data for the sharp morphology. The difference between $\mathrm{R}_{\mathrm{rms}}, \mathrm{R}_{\mathrm{sk}}$, and $\mathrm{R}_{\mathrm{ku}}$ for the two groups has already been discussed. On the contrary, the difference among the corresponding $\Delta(\mathrm{OSDI})$ is not confirmed by the $p$ values $(p=0.2173)$, mainly due to the anomaly of the results for the wearer 2 compared to the results for the other wearers of the same group.

\section{Conclusions}

A new Si-Hy material for disposable hydrophilic contact lenses treated with HA has been characterized. In general, the surface appears regular and flat. Before the wear, it is characterized by out-of-plane and sharp structures, with maximum height equal to about 10 nm. After 12 hours in saline solution, the main morphological characteristics are preserved. On the contrary, two typical morphologies are observed after 8 hours of wear. The former one (sharp-type) has a similar aspect as the unworn lenses, even if the height of the sharp peaks reaches about $30 \mathrm{~nm}$ and their number increases. The latter morphology (smooth-type) is totally different. It is characterized by troughs and bumpy structures. In this case, we 
attributed the changes to a loss of material, in agreement with the results of Goldberg et al. ${ }^{[32]}$ Typical low clinical OSDI values and $\Delta(\mathrm{OSDI})$ values have been obtained. Indications of different $\Delta(\mathrm{OSDI})$ have been found corresponding to the two morphologies and indication of correlation is found among $\Delta(\mathrm{OSDI})$ and the parameters which describe the morphology of the surface of the worn lenses, the worst clinical performances being correlated to the largest $\mathrm{R}_{\mathrm{rms}}$ and $\mathrm{R}_{\mathrm{sk}} \mathrm{AFM}$ parameters. This correlation can be explained as a consequence of the surface damage of the lenses due to surface abrasion of the smooth-type lenses and due to the changes of the surface parameters of the sharp-type lenses, even if the latter ones present a similar morphology as the unworn lenses.

Also for the contact-angle data, experimental evidence of correlation with the clinical performances has been found. As far as wettability is concerned, we have also found a strong effect due to the rinsing with deionized water, which determines a remarkable increase of the contact angle. In contrast to other explanations given in the literature, in our case this increase has been attributed to the removal of active hydrophilic agents from the lens itself, rather than to the blister solution. The slight further increase of the angle after wear is attributed to the modification of the surface morphology after wear and possible loss of moisture at the lens/air interface. Based on data reported in the literature, the increase of the contact angle is expected to be incompatible with lipid deposits on the surface.

The expected impacts of these results are: (i) the exploitation of this new low-roughness material for contact lenses, (ii) the further application of the well-known OSDI, whose modification after wear is here demonstrated to be correlated to the surface morphology and to the contact-angle measured on the worn lenses, (iii) the possible development of diagnostic methods for the eye conditions, based on the correlation with the surface properties of the worn lenses. 


\section{Acknowledgements}

We acknowledge the approval of all the involved subjects, Alessandro Filippo, Alessandro Borghesi, and Antonio Papagni for helpful discussions, and Peter Spearman for reading the paper.

\section{References}

[1] Gonzales-Meijome JM, Lopez-Alemany A, Almeida JB, Parafita MA, Refojo MF. Microscopic observations of superficial ultrastructure of unworn siloxane-hydrogel contact lenses by cryo-scanning electron microscopy. J Biomed Mater Res Part B: Appl Biomater. 2006;76B:419-423.

[2] Night\&Day and AirOptix (Ciba Vision).

[3] PureVision (Bausch+Lomb).

[4] NanoglossTM PremiO (Menicon).

[5] Acuvue Oasys (Johnson\&Johnson).

[6] Biofinity (CooperVision).

[7] Lin MC, Svitova TF. Contact lenses wettability in vitro: effects of surface-active ingredients. Optom Vis Sci. 2010;87:440-446.

[8] Polack FM, McNiece MT. The treatment of dry eyes with Na hyaluronate-Healon. Cornea 1982;1:133-136.

[9] Limberg MB, McCaa C, Kissling GE, Kaufman HE. Topical application of hyaluronic acid and chondroitin sulfate in the treatment of dry eyes. Am J Ophthalmol. 1987;103:194-197.

[10] Hamano T, Horimoto K, Lee M, Komemushi S. Sodium hyaluronate eye drops enhance tear film stability. Jpn J Ophthalmol. 1996;40:60-65. 
[11] Yokoi N, Komuro A, Nishida K, Kinoshita S. Effectiveness of hyaluronan on corneal epithelial barrier function in dry eye. Brit J Ophthalmol. 1997;81:533-536.

[12] Aragona P, Papa V, Micali A, Santocono M, Milazzo G. Long term treatment with sodium hyaluronate-containing artificial tear reduces ocular surface damage in patients with dry eye. Brit J Ophthalmol. 2002;86:181-184.

[13] Balazs EA. Viscoelastic properties of hyaluronan and its therapeutic use. In: Garg HG, Hales CA, editors. Chemistry and biology of hyaluronan. Amsterdam: Elsevier. $2004: 415$

[14] Falcone SJ, Palmeri D, Berg RA. Biomedical applications of hyaluronic acid. Polysaccharides Drug Deliv Pharm Appl (ACS Symp Ser) 2006;155-174.

[15] Johnson ME, Murphy PJ, Boulton M. Effectiveness of sodium hyaluronate eyedrops in the treatment of dry eye. Graefes Arch Clin Exp Ophthalmol. 2006;244:109-112.

[16] Kogan G, Solte’'s L, Stern R, Gemeiner P. Hyaluronic acid: a natural biopolymer with a broad range of biomedical and industrial applications. Biotechnol Lett. 2007;29:17-25.

[17] Baguet J, Somer F, Duc TM. Imaging surfaces of hydrophilic contact lenses with atomic force microscopy. Biomaterials. 1993;14:279-284.

[18] Teichroeb JH, Forrest JA, Ngai V, Martin JW, Jones L, Medley J. Imaging protein deposits on contact lens materials. Optom Vis Sci. 2008;85:1151-1164.

[19] Giraldez MJ, Serra C, Lira M, Elisabete M, Real Oliveira CD, Yebra-Pimentel E. Soft contact lens surface profile by atomic force microscopy. Optom Vis Sci. 2010;87:E475E481.

[20] Baguet J, Somer F, Claudon-Eyl V, Duc TM. Characterization of lachrymal component accumulation on worn soft contact lens surface by atomic force microscopy. Biomaterials. 1995;16:3-9. 
[21] Gonzalez-Meijome JM, Lopez-Alemany A, Almeida JB, Parafita MA, Refojo MF. Microscopic observation of unworn siloxane-hydrogel soft contact lenses by atomic force microscopy. J Biomed Mater Res Part B: Appl Biomater. 2006;76B:412-418.

[22] Lira M, Santos L, Yebra-Pimentel E, Elisabete M, Real Oliveira CD. Comparative study of silicone-hydrogel contact lenses surfaces before and after wear using atomic force microscopy. J Biomed Mater Res Part B: Appl Biomater. 2008; 85B: 361-367.

[23] (a) Ocular Surface Disease Index (OSDI), Allergan Inc, Irvine, California (Italian translation); (b) Schiffman RM, Christianson MD, Jacobsen G, Hirsch JD, Reis BL. Reliability and validity of the Ocular Surface Disease Index. Arch Ophthalmol. 2000;118:615-621.

[24] Sengor T, Kurna SA, Ozbay N, Ertek S, Aki S, Altun A. Contact lens-related dry eye and ocular surface changes with mapping technique in long-term soft silicone hydrogel contact lens wearers. Eur J Ophthalm. 2012; 22:S17-23.

[25] Berry M, Purslow C, Murphy PJ, Pult H. Contact lens materials, mucin fragmentation and relation to symptoms. Cornea 2012;31:770-776.

[26] Russo PA, Bouchard CS, Galasso JM. Extended-wear silicone hydrogel soft contact lenses in the management of moderate to severe dry eye signs and symptoms secondary to graft-versus-host disease. Eye Contact Lens 2007; 33:144-147.

[27] HA HyaCare (http://www.novozymes.com/en/investor/events-presentations/Document s/3_CMD_BB_HA_HAOK_FINAL.pdf), Novozymes.

[28] Fagnola M, Pagani MP, Maffioletti S, Tavazzi S, Papagni A. Hyaluronic acid in hydrophilic contact lenses: Spectroscopic investigation of the content and release in solution. Cont Lens Anterior Eye. 2009;32:108-112.

[29] Maldonado-Codina C, Morgan PB. In vitro water wettability of silicone hydrogel contact lenses determined using the sessile drop and captive bubble techniques. $\mathrm{J}$ Biomed Mater Res Part A. 2007;83A:496-502. 
[30] Lorentz H, Rogers R, Jones L. The impact of lipid on contact angle wettability. Optom Vis Sci. 2007;84:946-953.

[31] Gonzales-Meijome JM, Lopez-Alemany A, Almeida JB, Parafita MA. Surface AFM microscopy of unworn and worn samples of silicone hydrogel contact lenses. J Biomed Mater Res Part B: Appl Biomater. 2009;88B:75-82.

[32] Goldberg EP, Bhatia S, Enns JB. Hydrogel contact lens-corneal interactions: a new mechanism for deposit formation and corneal injury. CLAO J. 1997;23:243-248.

[33] Huh C, Mason SG. Effect on surface roughness on wettting. J Coll Interf Sci. 1977; 60:11-38.

[34] Synytska A, Ionov L, Minko S, Motornov M, Eichhorn KJ, Stamm M, Grundke K. Tuning wettability by controlled roughness and surface modification using core-shell particles. Polym Mater Sci Eng. 2004;90:624-625.

[35] Kubiak KJ, Wilson MCT, Mathia TG, Carval P. Wettability versus roughness of engineering surfaces. Proc 12th Intern Conf Metr \& Prop Eng Surf. Rzeszow, Poland. July 8-10, 2009;265-275. 
Figure captions

Fig. 1. AFM images with scan size $10 \mu \mathrm{m} \times 10 \mu \mathrm{m}$ (a) and $5 \mu \mathrm{m} \times 5 \mu \mathrm{m}$ (b) of the surface morphology of lenses taken from the blister and rinsed in deionized water $\left(\mathrm{CL}_{\text {rins }}\right)$.

Fig. 2. AFM images with scan size $10 \mu \mathrm{m} \times 10 \mu \mathrm{m}$ (a) and $5 \mu \mathrm{m} \times 5 \mu \mathrm{m}$ (b) of the surface morphology of lenses preserved 12 hours in saline solution and rinsed with deionized water $\left(\mathrm{CL}_{\mathrm{ss}}\right)$.

Fig. 3. AFM images with scan size $10 \mu \mathrm{m} \times 10 \mu \mathrm{m}$ (a) and $5 \mu \mathrm{m} \times 5 \mu \mathrm{m}$ (b) of the sharp-type surface morphology of lenses worn for 8 hours, preserved 12 hours in saline solution, and rinsed in deionized water $\left(\mathrm{CL}_{\text {worn }}\right)$.

Fig. 4. AFM images with scan size $10 \mu \mathrm{m} \times 10 \mu \mathrm{m}$ (a) and $5 \mu \mathrm{m} \times 5 \mu \mathrm{m}$ (b) of the smoothtype surface morphology of lenses worn for 8 hours, preserved 12 hours in saline solution, and rinsed in deionized water $\left(\mathrm{CL}_{\text {worn }}\right)$.

Fig. 5. 3D AFM plots of lenses taken from the blister and rinsed in deionized water $\left(\mathrm{CL}_{\text {rins }}\right)$ (first panel) and worn for 8 hours, preserved 12 hours in saline solution, and rinsed in deionized water ( $\mathrm{CL}_{\text {worn }}$, second panel: smooth-type, third panel: sharp type).

Fig. 6. Exemplificative water droplet images captured $10 \mathrm{~s}$ after droplet deposition on the four different types of lenses: (a) lens directly taken from the blister $\left(\mathrm{CL}_{\mathrm{bl}}\right)$, (b) lens taken from the blister and rinsed in deionized water $\left(\mathrm{CL}_{\text {rins }}\right)$, (c) lens preserved 12 hours in saline solution and rinsed with deionized water $\left(\mathrm{CL}_{\mathrm{ss}}\right)$, and (d) lens worn for 8 hours, preserved 12 hours in saline solution, and rinsed in deionized water $\left(\mathrm{CL}_{\mathrm{worn}}\right)$. 
Fig. 7. Roughness, skewness, and kurtosis deduced from AFM analysis of worn contact lenses as a function of the change of the OSDI. Black diamonds correspond to the smooth-type morphology. Red squares correspond to the sharp-type morphology. The labels correspond to the subject identification numbers (repeated twice when data for the right and left eyes are available, repeated more than twice when the wear has been repeated at the distance of weeks). Continuous lines indicate the results of the linear fitting of the data. The corresponding $\mathrm{R}$ and $p_{\mathrm{c}}$ values are also given in each panel.

Fig 8. Measured contact angle of worn contact lenses as a function of the change of the OSDI. A continuous line indicates the result of the linear fitting of the data. 